\title{
Inertia and propulsion in general relativity: a reply to rodal
}

\author{
James F. Woodward' ${ }^{1}$
}

Received: 8 July 2019 / Accepted: 16 December 2019 / Published online: 1 February 2020

(c) The Author(s) 2020

\begin{abstract}
In a recent issue of this journal, Rodal (Gen Relativ Gravit 51:61-84, 2019) has written an extensive critique of a novel propulsion scheme devised nearly 30 years ago (Woodward in Found Phys Lett 6:1-39, 1995; Making starships and stargates: the science of interstellar transport and absurdly benign wormholes, Springer, New York, 2012). That scheme depends on the production of rest mass fluctuations, driven in special systems, that arise when the systems are accelerated, through the interaction of the systems with the field that produces inertial forces. Following Einstein, the field that produces inertial forces is taken to be the gravitational field due chiefly to the most distant matter (everything that gravitates) in the universe. Such rest mass fluctuations in local accelerating objects, predicted using the relativistic Newtonian approximation to general relativity, are now called "Mach effects" since they depend on inertial forces in fact being gravitational forces as Ernst Mach hinted might be the case. Rodal claims that such rest mass fluctuations with significant magnitude, save in the vicinity of black holes, are not to be found in general relativity notwithstanding that they appear in the relativistic Newtonian approximation. Rodal is mistaken in this claim, as I have already explained at some length (Woodward in J Br Interplanet Soc 70:354-364, 2018). Here I recapitulate some of that material and expand some material with specific reference to his now published claims.
\end{abstract}

Keywords Gravitational origin of inertia $\cdot$ Mach's principle $\cdot$ Propulsion

\section{Introduction}

The plan of this note is as follows. First, the key features of the relativistic Newtonian approximation to general relativity are identified so there can be no mistake about how large rest mass fluctuations come about, or whether they really are a matter of general relativity. Next, passages of Rodal's [1] essay are reproduced and

James F. Woodward

jwoodward@fullerton.edu

1 Department of Physics, California State University Fullerton, Fullerton, CA 92834, USA 
errors therein are identified. Finally, since these errors arise from a misunderstanding of Carl Brans' "spectator matter" argument, that argument is briefly revisited to clarify its meaning. In particular, it is noted that the "spectator matter" argument demands that the "locally measured" Newtonian gravitational potential be an invariant that must be proportional to the square of the vacuum speed of light to correctly account for inertia and Newtonian mechanics (with relativistic corrections of course) when and wherever it is measured if the Equivalence Principle is not to be violated. This ensures that the Newtonian potential everywhere/when is sufficient to produce the rest mass fluctuations upon which the novel propulsion scheme depends.

\section{Rest mass fluctuations}

If your interest in gravity and inertia stems from a desire to figure out how to get around spacetime quickly, you will likely discover that you must understand gravity and inertia if your hope for success is realistic. The reason why is simple. Two requirements must be satisfied: One, you must create a simple mechanical device in which rest mass fluctuations can be induced by local electrical and/or mechanical actions. For example, a periodic rest mass fluctuation can be produced by periodically charging and discharging a capacitor. The resulting $E / c^{2}$ mass fluctuations, however, are minuscule. So, two, the rest mass fluctuations in the device must interact with large gravitational forces in such a way that the interaction of the rest mass fluctuations and inertio-gravitational field induce large transient sources of the field. That is, the interaction with the inertio-gravitational field must effectively amplify the otherwise miniscule rest mass fluctuations by orders of magnitude. The only local gravitational forces and field large enough to be of any use are inertial forcesof course, assuming that inertial forces are gravitational in origin.

Although almost nothing on inertia as part of the gravitational interaction is to be found in textbooks on general relativity [2], over the past 30 years or so increasing attention has been paid to the issue. [3-5] For the moment, I note that if inertia is actually a gravitational effect (as Einstein insisted), then any interaction involving inertia is a zeroth order effect as inertial effects, being due to the cumulative effect of the bulk of the matter in the universe, are many orders of magnitude larger than any other gravitational effect, save in immediate proximity to the horizons of black holes. We will return to the issue of the origin of inertia after first considering the other technical issue-transient rest mass effects-that must be dealt with in any realistic grav/inertial propulsion scheme.

If you ask how inertial forces might be employed for propulsion, not much with any realistic promise will likely come quickly to mind. The conservation laws of Newtonian physics seem to preclude all plausible schemes. However, 30 years ago, I was confronted with the realization that a relativistic Newtonian approximation gravitational field equation constructed by Luchak $[6]^{1}$ hinted at the possibility that

\footnotetext{
1 The field equation is: $\nabla \cdot \mathbf{F}+\frac{1}{c} \frac{\partial q}{\partial t}=-4 \pi \rho$ with $\nabla q+\frac{1}{c} \frac{\partial \mathbf{F}}{\partial t}=0$ and $\nabla \times \mathbf{F}=0 . q$ is the rate at which the field does work on its local sources and $\rho$ the source density. The time-dependent term in the first of these equations is where rest mass fluctuations are to be sought.
} 
induction of transient sources of gravity/inertia might be possible. A path to propulsion involving a transient interaction of a small local system with the grav/inertial field of the universe wherein mass and momentum transfer between the local system and the field might work opened up. The field that causes inertia is many orders of magnitude larger than the contributions to the field due to local sources. And transient effects scale inversely with some power of the duration of the transient, so even small, local effects might prove adequate to the purpose if done quickly enough in such a large field.

There were no standard references to assess the merits of this conjecture [7, Ch. 3]. As a substitute for a check with standard references, I reconstructed Luchak's relativistic Newtonian approximation field equation from first principles to convince myself that it was reasonably applicable to the inertial forces experienced by local objects subjected to proper accelerations (and took the calculation to friends expert in general relativity for scrutiny). Note that nowhere in this process is the assumption made that the theory of gravity and inertia deviates in any way from general relativity - provided that Einstein was right about the gravitational origin of inertia. Indeed, general relativity is required to get the local strength of the gravitational field due to cosmic matter. Magical properties of the quantum vacuum are not assumed, neither is some speculative unified field theory invoked. No cavorite. It's all just standard physics tasked to answering an unconventional question.

I will not rederive in detail the relativistic Newtonian approximation gravitational field equation here. That derivation is found in a number of papers starting with [8], and most completely in chapter 3 of [7]. Suffice it to say that rest mass fluctuations have their origin in the definition of four-forces in special relativity. The four-force definition includes a term that is the derivative with respect to proper time of the time-like component of the four-momentum, $m c$. That is, $c d m / d t$. Using $E=m c^{2}$, this can be written as $(1 / c) d E / d t$. When this shows up in the field equation, it is easily identified as the rate that the field does work on its local sources (per unit volume of course as the field equation is expressed in terms of densities).

The local sources of the inertio-gravitational field can be obtained in the usual way: taking the four-divergence of the field. When this is done, the time-dependent term in the field equation introduced by special relativity becomes $\frac{1}{c^{2}} \frac{\partial^{2} E}{\partial t^{2}}$. The spacelike part of the divergence is just the Laplacian of the Newtonian potential. So, to put the field equation into standard differential form - a classical wave equation- $-\mathrm{a}$ way to extract and isolate a second time derivative of the potential from the second time derivative of the energy density must be found. This entails a short digression into the particulars of how gravity and inertia work in general relativity. Since this issue has been one of great contention and often turgid and unconvincing arguments in the community of general relativists over the decades and to the present, I will use Sciama's [9] argument on the origin of inertia to lay out the important issues because of its great simplicity and clarity. It is only a vector approximation to the tensor general relativistic treatment. But since the term that leads to the gravitational 
induction of inertial forces, absent in scalar gravity theories, appears in vector gravity theory, it is good enough for our purpose. ${ }^{2}$

Consider Sciama's "gravelectric" field equation:

$$
\mathbf{E}=-\nabla \phi-\frac{1}{c} \frac{\partial \mathbf{A}}{\partial t}
$$

$\mathbf{E}$ is the gravelectric field strength, $\phi$ the total Newtonian scalar potential and $\mathbf{A}$ the gravimagnetic vector potential. The values of the potentials are obtained by integrating over all matter, and matter currents respectively out the past light cone to the past particle horizon. Sciama initially intended his vector theory of gravity to be an alternative to general relativity. He also broke up the scalar potential into local $\left[\phi_{l}\right]$ and cosmic $[\Phi]$ parts, as does Rodal. Nonetheless, it is the total Newtonian potential that figures into this equation. As Sciama noted, the integration over matter currents to get $\mathbf{A}$ can be reformulated as an integration over matter distribution alone. If you are moving with some velocity $\mathbf{v}$ with respect to your mean cosmic rest frame and you want to calculate the mass current contributions to get the value of A, all you have to do is assume yourself at rest with the universe apparently moving rigidly past you with velocity- $-\mathbf{v}$. This means that the matter currents, $m \mathbf{v}$, to be integrated over can have the velocity removed from the integration as $\mathbf{v}$ is the same everywhere, leaving only the effect of an integration over the matter sources-which returns an integration for the Newtonian scalar potential. Note that retardation of propagating fields is automatically accounted for in this calculation.

In light of these considerations, when a body is accelerated, the inertial force per unit mass the body experiences is:

$$
\mathbf{E}=-\frac{1}{c} \frac{\partial \mathbf{A}}{\partial t}=-\frac{\phi}{c^{2}} \frac{d \mathbf{v}}{d t}=-\frac{\left(\phi_{l}+\Phi\right)}{c^{2}} \frac{d \mathbf{v}}{d t}
$$

and if $\phi=c^{2}$, then $\mathbf{E} m$ is the inertial force communicated through the accelerating body with mass $m$ to the accelerating agent. The same result with a factor of 4 is recovered in the tensor equivalent of this calculation.

Promising though this may appear, naively, there seem to be problems. In special relativity $c$ is a simple constant. In general relativity $c$ becomes a "locally measured invariant" because although the same number for the magnitude of $c$ is obtained in all local tests, the value of $c$ in non-local tests may differ from the local value. The commonplace example of this behavior is the speed of light near the event horizon

\footnotetext{
2 The "Faradayan" gravitomagnetic induction term in the equation of motion arises from the time derivative of the vector potential and is, in Sciama's view, the cause of inertial forces. General relativity has a corresponding gravitomagnetic term that with a suitable coefficient performs the same function. It is now fashionable to claim that in the weak field, time dependent, non-relativistic limit, this term can be made to vanish by a gauge condition - and that this can be generalized to all circumstances [5, chapter 4]. This argument, however, depends on a particular choice of coordinates (deDonder), not a gauge condition. As Ken Nordtvedt showed in 1988 [10], while the gravitomagnetic vector potential contribution to, for example, orbit calculations and other local astrophysical situations can be made to vanish with felicitous choice of coordinates, other coordinate choices, even at lowest order, require that terms involving the vector potential be included to get sensible results.
} 
of a black hole-as determined by a distant observer-tending to zero as the horizon is approached by the light. While $c$ is a locally measured invariant, and $G$ may be too, $R$ at least in an expanding universe is a function of time. Since $\phi / c^{2}$ must be a constant equal to 1 in the vector approximation if Newton's third law is to be valid everywhere/when, this is a non-trivial problem. Which we will return to after considering Rodal's critique.

Armed with our knowledge that inductive inertia requires that the coefficient of $d \mathbf{v} / d t$ in the $d \mathbf{A} / d t$ term in the gravelectic field equation (and its counterpart in general relativity) be one, we can use $\phi=c^{2}$ to accomplish the task of recovering the d'Alembertian of $\phi$. In addition to a term in the second time derivative of the potential that completes the d'Alembert wave equation for the potential, several timedependent terms are recovered that must be transferred to the source (right-hand) side of the equation. These are the terms that are designated "Mach effects" for they depend for their existence almost exclusively on the potential that arises from the existence of matter at cosmic distances from us, local contributions being negligible. The equation that results from these operations [7, 8, p. 93, eq 4.1] is:

$$
\nabla^{2} \phi-\frac{1}{c^{2}} \frac{\partial^{2} \phi}{\partial t^{2}}=4 \pi G \rho_{o}+\frac{\phi}{\rho_{o} c^{4}} \frac{\partial^{2} E_{o}}{\partial t^{2}}-\left(\frac{\phi}{\rho_{o} c^{4}}\right)^{2}\left(\frac{\partial E_{o}}{\partial t}\right)^{2}-\frac{1}{c^{4}}\left(\frac{\partial \phi}{\partial t}\right)^{2}
$$

where $\rho_{\mathrm{o}}$ and $E_{\mathrm{o}}$ are the proper mass and energy densities at the point where the divergence is taken. Since the time-dependent terms on the right hand (source) side of Eq. (3) originate on the left hand [field] side of the equation, they do not carry the coefficient $4 \pi G$. To be treated as massive sources (and multiplied by $4 \pi G$ like $\rho_{\mathrm{o}}$ in the first term on the right-hand side of Eq. (3)) they must be multiplied by the factor $(1 / 4 \pi \mathrm{G})$. Note that this procedure for recovering a wave equation to identify the time-dependent local sources of the gravitational field is a general relativistic procedure since it depends on inertia being a gravitational effect due chiefly to distant matter as Einstein insisted giving $\phi=c^{2}$ must be the case in general relativity.

\section{Rodal's critique of rest mass transients in general relativity}

Rather than synopsize Rodal's argument against lowest order rest mass fluctuations as general relativistic effects, I will quote the gist of Rodal's argument in his own words and add comments. In the introduction we find:

Section 2 shows that Einstein's theory [general relativity] has a similar (to Woodward's theory) term, but it is much smaller in magnitude ( $3^{\text {rd }}$ order postNewtonian) because its prefactor is the local gravitational potential which (unless a spaceship were to be near a black hole or neutron star) is infinitesimal. Therefore it is irrelevant for practical space propulsion (for this application it does not matter whether this force is physical or is the result of a coordinate effect). [Emphasis added.] 
The parenthetical reference to "Woodward's theory" is misleading. There is no "Woodward's theory". There is general relativity applied in a calculation at relativistic Newtonian approximation to a specific set of circumstances. The emphasized text refers to Carl Brans' "spectator matter" argument. Brans' argument plays a critical role in getting inertia in general relativity right, and fixes it in such a way that the "locally measured" value of the Newtonian potential is always dominated by the contribution of distant matter, no matter the order of a post-Newtonian perturbation expansion where it occurs. Brans' argument implies that the locally measured value of the Newtonian potential, if non-zero, must be a scalar invariant that makes inertial forces equal and opposite to applied non-gravitational accelerating forces [3]. Rodal ignores this fact, preferring to adopt the interpretation given by Brans [11] wherein the effective local Newtonian potential has no contributions from cosmological matter and is therefore essentially zero. Given the state of cosmological knowledge in the '70s, this assumption was reasonable then. It is not reasonable now. The rest of the introduction deals with matters that turn out to be irrelevant, ending with, "In summary, this effect appears too small for practical space travel application (unless the spaceship is near a black hole or a neutron star)."

In Sect. 2 we find:

Woodward derived, for a system with very weak gravitational field and mass $m=\int_{V} \rho d V$ varying with time the existence of a gravitational field term proportional to the second time derivative of the fluctuating mass....

This assertion by Rodal is incorrect. The scalar potential in the "Woodward" derivation-Eq. (3) above-is everywhere the total scalar Newtonian potential, for a process involving inertial forces is being described, and those forces arise chiefly from distant matter sources, not nearby local sources that produce minuscule contributions to the field by comparison. The local fluctuating mass does not arise from the action of some local gravitational potential. It arises as a special relativistic effect from the derivative of the time-like part of the four-momentum of the local object being that is being accelerated by a non-gravitational force. That minuscule special relativistic mass fluctuation is effectively vastly amplified by the action of the total, not just local, gravitational field on the accelerating object.

Rodal continues,

Woodward's equation has the D'Alembertian of the weak gravitational potential $\phi=-G m / r$ of the local mass source (where $r$ is the radius of the spherically symmetric weak field), on the left-hand side of the field equation and, on the right-hand side, in addition to the usual matter source term $4 \pi G \rho$, an additional source term $\frac{\Phi+\phi_{l}}{m c^{2}} \frac{\partial^{2} m}{\partial t^{2}} \approx \frac{\Phi}{m c^{2}} \frac{\partial^{2} m}{\partial t^{2}}$ where $\Phi=G M / R$ is the scalar potential of the whole universe (with the universe's effective gravitational mass $M$ and effective radius $R$ ), satisfying Sciama's condition $\left(\Phi+\phi_{l}\right) / c^{2}=1 \approx \Phi / c^{2}$ since $|\Phi| \gg\rangle\left|\phi_{l}\right|$.

Rodal's justification for making his assertion about the potential seems to be that the large potential due to distant sources can be treated as a constant, so space 
and time derivatives of that part of the potential vanish. But no part of the potential involved in the interaction can be treated as constant. As we will see in the next section where the work of Carl Brans is discussed, the locally measured Newtonian potential is a "locally measured invariant"-like the vacuum speed of light - and since locally measured invariants have non-local values that may differ from the locally measured value, space and time derivatives of locally measured invariants do not in general vanish. The potential due to all sources, near and far, by Brans' argument is a locally measured invariant, so no sources of the field contribute in a way that they can be treated as constants, and the derivatives do not vanish for any part of the field. Thus, the potential acted upon by the d'Alembertian in Eq. (3) is not the "local" potential in the sense that the potential is due only to local sources. It is the locally measured invariant total Newtonian potential with which a small, local accelerating mass with varying internal energy interacts. This is also true wherever the Newtonian potential shows up in calculations at any arbitrary "order" of any local perturbation since it is a locally measured invariant.

Rodal then invokes a treatment of general relativity developed by Landau and Lifschitz using the deDonder coordinate condition and the Minkowski metric of special relativity. They, Landau and Lifschitz, constructed an exact field equation with this choice of coordinates and metric that has the form of the d'Alembertian of the field is equal to the usual sources involving the stress-energy-momentum tensor and the pseudo tensor of the field, but also includes a term involving field quantities with the "prefactor" [coefficient] $c^{2} / 4 \pi G$. (Poisson and Will, chapter 6 is helpful here. [12]) According to Rodal,

In this harmonic gauge, the exact field equation takes the Landau-Lifschitz form:

$$
\square h^{\alpha \beta}=\frac{8 \pi G}{c^{2}}\left((-g)\left(T^{\alpha \beta}+t_{L L}^{\alpha \beta}\right)+\frac{c^{2}}{4 \pi G}\left(\left(h^{\alpha \mu}\right)_{, \nu}\left(h^{\beta \nu}\right)_{, \mu}-h^{\mu \nu}\left(h^{\alpha \beta}\right)_{, \mu, \nu}\right)\right)
$$

Where $c^{2}$ in the denominator of the factor $8 \pi G / c^{2}$ corresponds to a definition of the stress-energy-momentum tensor $T_{\mu}^{\nu}$ mixed components with units of mass density (otherwise, using units of energy density the denominator would be $\left.c^{4}\right)$.

is the four-dimensional Laplacian on Minkowski spacetime, that is, the d'Alembertian. Rodal goes on to enumerate several remarkable properties and limitations of Landau and Lifschitz's above equation. The arresting term in this equation, of course, is the last term, the second derivative of $h^{\alpha \beta}$ with coefficient $c^{2} / 4 \pi G$. Evidently, this term is the counterpart of the leading time-dependent source term of the relativistic Newtonian approximation used in Mach effects. Rodal, after showing that this term leads to a second time-derivative of a variable mass then remarks, "This term, dependent on the second time derivative of a variable mass, is much smaller in magnitude than the term derived by Woodward, because its prefactor is the local potential $\phi_{l}$ instead of the universe's potential $\Phi$." Note that the local potential here is NOT the potential due to the fluctuating 
mass. That is, $h^{\alpha \beta}$. It is, allegedly, the potential of some nearby spectator mass source-that is, $h^{\mu \nu}\left[=\phi_{l}\right.$ for $\left.\mu=v=0\right]$ in Eq. (4) above. This is why Rodal remarks that mass fluctuation effects in the vicinity of a black hole are large.

Again, Rodal's mistake is to take the locally measured potential to be due only to nearby sources. If, however, the locally measured Newtonian gravitational potential satisfies Sciama's sum for inertia that includes cosmological sources, as it must to account for inertia as a gravitational effect, then Rodal's analysis shows that mass fluctuations can be large enough for propulsive purposes irrespective of the post Newtonian order at which the fluctuations appear. Why? Because the Newtonian potential, local or otherwise, by definition, is the sum of the contributions of all matter sources integrated out the past light cone to the past particle horizon. And this potential is a multiplier of the miniscule relativistic $\mathrm{E} / \mathrm{c}^{2}$ mass fluctuation that interacts with the local gravitational field, vastly amplifying the mass fluctuation. To initiate this multiplier effect, however, the fluctuating mass must be given proper acceleration to make manifest the gravitational field that causes inertial effects. You might think the distant sources unimportant. But while their influence decreases as $1 / r$, their number increases as $r^{2}$. Nonetheless, you might argue that the cosmic contributions to $\phi$ are effectively constant, without derivatives, and so ignorable. If general relativity were a gauge theory, you might be able to get rid of the influence of distant sources by some clever choice of gauge. But general relativity is not a gauge theory. And all sources of $\phi$ out to the past particle horizon must be taken into account when computing the local potential.

Rodal continues, "Furthermore, the above harmonic-coordinate-dependent derivation does not settle whether this term is the result of a coordinate effect and hence whether it has physically detectable consequences." This comment about a "coordinate effect" is an oblique reference to the work already mentioned by Carl Brans, to which we now turn.

\section{Carl Brans and the origin of inertia}

Dennis Sciama's paper "On the Origin of Inertia" focused attention on the issue of inertia in gravitation theory. As a vector theory, Sciama's work was at best an approximation to general relativity. So, when Carl Brans passed his qualifying exams at Princeton in 1958, his doctoral supervisor, Robert Dicke, set him to investigating whether Mach's principle could be incorporated into tensor general relativity. Reviewing Einstein's comments on Machian inertia made in lectures at Princeton in 1921 [13] Brans noted a problem with Einstein's argument. Brans wrote in 1977:

Over the years, many and varied expressions of Mach's principle have been proposed, making it one of the most elusive concepts in physics. However, it seems clear that Einstein intended to show that locally measured inertial-mass values are gravitationally coupled to the mass distribution in the universe in 
his theory. For convenience I repeat the first order geodesic equations given by Einstein to support his argument:

$$
\begin{aligned}
& (d / d t)[(l+\bar{\sigma})]=\nabla \bar{\sigma}+\partial \mathbf{A} / \partial l+\nabla \times(\mathbf{A} \times \mathbf{v}), \\
& \bar{\sigma}=(\kappa / 8 \pi) \int(\sigma / r) d V_{o}, \\
& \mathbf{A}=(\kappa / 2 \pi) \int(\sigma d \mathbf{x} / d l) r^{-1} d V_{o} .
\end{aligned}
$$

Here $\sigma$ is the source-mass density while $l$ is coordinate time and $\mathbf{v}$ is coordinate velocity of a test particle. Einstein's claim is that "The inertial mass is proportional to $(l+\bar{\sigma})$, and therefore increases when ponderable masses approach the test body.

Brans pointed out that having the masses of local objects depend on their gravitational potential energies acquired by interaction with nearby objects cannot be right. If it were true, then for example the electric charge to mass ratios of elementary particles would depend on the presence of nearby matter, and gravity could be discriminated from accelerations without having to check for the presence of spectator matter - a violation of the Equivalence Principle. From this, Brans inferred that

... global, i.e., nontidal, gravitational fields are completely invisible in such local standard measurements of inertial mass, contrary to Einstein's claim.... Einstein ought to have normalized his local space-time measurements to inertial frames, in which the metric has been transformed approximately to the standard Minkowski values, and for which distant-matter contributions are not present. [Emphasis added.]

The "coordinate condition" mentioned with respect to Brans' work is that the coordinates be compatible with the assumed approximate Minkowski metric applicable in small regions of spacetime. Since the absence of gravity is presupposed for Minkowski spacetime, this amounts to the assumption that the Newtonian potential due to exterior matter in such small regions of spacetime is effectively everywhere/ when equal to zero. Where there is effectively no gravity there can be no gravitational induction of inertia. Accordingly, it would seem that the spectator matter argument makes Machian inertia incompatible with general relativity.

Brans' "spectator matter" argument is, of course, absolutely correct. Having the rest masses of objects depend on their gravitational potential energies due to nearby "spectator" matter is a clear violation of the Equivalence Principle. But to extend that inference to the assumption that inertia and inertial forces are not gravitationally induced is a mistake.

If we are to recover gravitationally induced inertial forces in a small shielded laboratory, from both Sciama's and Einstein's (as quoted by Brans above) calculations we see-as a consequence of Sciama's argument that the derivative of the vector potential is just $\phi / c^{2}$ times the acceleration-that $\phi$ must be equal to $c^{2}$ throughout the lab so that the coefficient of the acceleration is a constant equal to one. Minkowski spacetime does not do this for us since $\phi$ is by definition zero. But 
essentially flat spacetime is not necessarily Minkowskian in general relativity and our universe. So, the question at this point is: Is there a sufficiently flat spacetime that can be substituted for Minkowski spacetime that meets Einstein's requirement of the gravitational induction of inertia without messing up important calculations?

Happily, the answer to this question is yes. It has been known for decades that the " $k=0$ " solution for the Friedmann-Robertson-Walker (FRW) cosmological field equations is spatially flat, so in sufficiently small regions of spacetime where we can take spacetime as essentially flat (irrespective of whether non-vanishing curvature may be present), we can assume that the physical conditions that generate FRW, $\mathrm{k}=0$ conditions are present. What are the conditions that produce at least approximately flat spacetime? "Critical" cosmic matter density at cosmic scale (remember, distant matter is the alleged cause of inertia, so having the influence of that matter contribute is to be expected) which yields $\Omega=1$ that in turn leads to $\phi=c^{2}$, where $\phi=G M / R$ with $G$ being Newton's constant of gravitation, $M$ the effective mass of the universe, and $R$ being its effective radius-Sciama's sum for inertia. A noteworthy feature of the FRW, $\mathrm{k}=0$ cosmology is that it is epoch independent. So the special relationship between $\phi$ and $c$ is automatically satisfied as it is time-independent.

The epoch independence of $\phi$, and thus $\phi / c^{2}$, in FRW, $\mathrm{k}=0$ cosmology might be viewed as just a happy coincidence. But the real meaning of Brans' spectator matter argument is not that there are no inertial effects that might be attributed to gravity in small isolated labs. It is that if there are inertial effects, inertial forces in particular, in isolated labs, then the Newtonian gravitational potential involved must be a locally measured scalar invariant_with a magnitude that accounts for the origin of inertia in material objects and inertia forces as gravitational actions. If such a potential can arise in general relativity, then inertia is a gravitational phenomenon in that theory. This is precisely what happens in the FRW, $\mathrm{k}=0$ cosmology, and the cosmological part of the integration for the Newtonian potential dominates by orders of magnitude the contributions of all other sources save in the vicinity of black holes. The straight-forward inference is that Brans' spectator matter argument shows that the total Newtonian potential in our mostly spatially nearly flat reality is a locally measured scalar invariant; the counterpart for gravitation to the vacuum speed of light for electrodynamics. And they are related too, one being (at least up to a factor of the order of one) the square of the other.

The locally measured invariant status that $\phi$ acquires as a consequence of Brans' spectator matter argument locks inertia into general relativity as a gravitational effect-making it one of the most consequential developments in general relativity of the past century. How? Well, all we need to avoid violating the Equivalence Principle is for $\phi$ to be a locally measured invariant equal to $c^{2}$ in FRW, $\mathrm{k}=0$ cosmology to get inductive inertial effects right. FRW, $\mathrm{k}= \pm 1$ do not have epoch independent critical comic matter density, so $\phi$ in those models is not a locally measured invariant, and the coefficients of the induction terms are not constants and Newton's third law is not universally valid. Brans' spectator matter argument singles out a unique FRW cosmology as compatible with general relativity and Newtonian physics.

In the matter of Rodal's critique of Mach effects, the locally measured invariance of $\phi$ ensures that the potential that multiplies the second time-derivative of the local restmass in Eq. (4) above is NOT the "local", small $\phi$. That is, $\phi_{l}$. It is the locally 
measured invariant value of $\phi$. That is, $\phi=c^{2}$. One need not be in the vicinity of a black hole to propulsively interact with a gravitational field of sufficient strength to get around spacetime quicky.

Common sense suggests that any correct theory of gravity should have local dynamical consequences arising from the distant matter in the universe. After all, its influence is only matched by local sources in the neighborhood of black holes. We live in the presence of spacetime/gravity of enormous strength. But because we intuitively associate spatial flatness with the absence of gravity, we intuitively reject the idea that a gravity field with a strength comparable to that near the horizon of a black hole is our reality. That is why "Mach's principle" has been the subject of so much debate in the past century. The really interesting question is can we get around in it quickly? That is a question to be answered by experiment.

\section{Conclusion}

The gravitational origin of inertia and inertial forces has underlain the Mach effects project from its inception in the fall of 1989. Serious discussion of the viability of the proposal started in email exchanges with Paul Zielinski and Jack Sarfatti in 2012 through 2014 - about the time that my colleague Heidi Fearn joined the project, with whom I have had ongoing discussions of the issues involved. In 2015 and 2016 Lance Williams and I frequently discussed gravitational inertia, mostly telephonically. Other less formal, episodic discussions have taken place over the years with, for example, Olivier Costa de Beauregard, Peter Milonni, John Cramer, Keith Wanser, and Raymond Chiao. Others have helped in significant ways. Gary Hudson, Nembo Buldrini, David Jenkins, Paul March, David Mathes, Jim Peoples, John Brandenburg, Chip Akins, Marshall Eubanks, Greg Meholic, Martin Tajmar, to name but a few. Nick Herbert suggested an interesting voltage scaling test carried out several years ago and repeated a year ago successfully.

The role of inertia in general relativity has been contentious from the outset. Indeed, Tullio Levi-Civita had to point out to Einstein that the affine connection (which carries the inertial part of the gravitational interaction) is more fundamental than the curvature of spacetime (widely thought to be the marker of gravity). The arguments, however, have been restricted to the general relativity community and "academic" until quite recently. For example, a decade and more ago, when the Higgs interaction was widely hailed as the "origin of mass" (mass being the measure of inertia), no one even bothered to mention that Einstein took gravity to be the origin of inertia and that the topic had been long and at times hotly debated in the general relativity community. Rodal's dismissive critique of Mach effects falls squarely within this tradition. A year or so ago, however, he sent me an apposite Feynman quote: "It doesn't matter how beautiful your theory is. It doesn't matter how smart you are. If it doesn't agree with experiment, it's wrong." Heidi and I have an apparatus running in a lab we share that says that the locally measured invariant nature of the Newtonian potential that follows from Carl Brans' spectator matter argument involving the Equivalence Principle is correct. Exhaustive tests show that 
the observed forces produced by small Mach effect thrusters (METs), or, as Heidi prefers, Mach effect gravity assist (MEGA) drives cannot be ascribed to plausible conventional causes.

However that experimental work eventually turns out, it is worth remarking that until inertia is correctly understood as an integral part of the gravitational interaction, no one is going anywhere very fast—and maybe not even then.

Acknowledgements This work was supported in part by a Phase 2 NASA National Innovative Advanced Concepts (NIAC) grant, "Mach effects for in space propulsion: Interstellar mission," Grant NNX17AJ78G. In anticipation of critiques like Rodal's, much of the material in Sects. 2 and 4 here formed a short presentation on 30 April 2019 to the NIAC mid-term review team at CSUF. Dan Kennefick, who recently joined the NIAC Grant project, was kind enough to look over this note (and [3]) to catch any egregiously bad mistakes.

Open Access This article is licensed under a Creative Commons Attribution 4.0 International License, which permits use, sharing, adaptation, distribution and reproduction in any medium or format, as long as you give appropriate credit to the original author(s) and the source, provide a link to the Creative Commons licence, and indicate if changes were made. The images or other third party material in this article are included in the article's Creative Commons licence, unless indicated otherwise in a credit line to the material. If material is not included in the article's Creative Commons licence and your intended use is not permitted by statutory regulation or exceeds the permitted use, you will need to obtain permission directly from the copyright holder. To view a copy of this licence, visit http://creativecommons.org/licen ses/by/4.0/.

\section{References}

1. Rodal, J.J.A.: A Machian wave effect in conformal, scalar-tensor gravitational theory. Gen. Relativ. Gravit. 51, 61-84 (2019)

2. Stachel, J.: The hole argument and some physical ad philosophical implications. Living Rev. Relat. 17, 1-66 (2014)

3. Woodward, J.F.: Einstein, Mach's principle, and the unification of gravity and inertia. J. Br. Interplanet. Soc. 70, 354-364 (2018). (actually, the 2017 volume published in 2018)

4. Ciufolini, I., Wheeler, J.A.: Gravitation and Inertia. Princeton University Press, Princeton (1995)

5. Pfister, H., King, M.: Inertia and Gravitation. Springer, New York (2015)

6. Luchak, G.: A fundamental theory of the magnetism of rotating bodies. Can. J. Phys. 29, 470-479 (1951)

7. Woodward, J.F.: Making Starships and Stargates: The Science of Interstellar Transport and Absurdly Benign Wormholes. Springer, New York (2012)

8. Woodward, J.F.: Making the universe safe for historians: time travel and the laws of physics. Found. Phys. Lett. 6, 1-39 (1995)

9. Sciama, D.: On the origin of inertia. MNRAS 34, 34-42 (1953)

10. Nordtvedt, K.: Existence of the gravitomagnetic interaction. Int. J. Theor. Phys. 27, 1395-1404 (1988)

11. Brans, C.H.: Absence of inertial induction in general relativity. Phys. Rev. Lett. 39, 856 (1977)

12. Poisson, E., Will, C.H.: Gravity. Cambridge University Press, Cambridge (2014)

13. Einstein, A.: The Meaning of Relativity, 5th edn. Princeton University Press, Princeton (1955)

Publisher's Note Springer Nature remains neutral with regard to jurisdictional claims in published maps and institutional affiliations. 